\title{
From Modelica Models to Fault Diagnosis in Air Handling Units
}

\author{
Raymond Sterling ${ }^{1}$, Peter Struss ${ }^{2}$, Jesús Febres ${ }^{1}$, Umbreen Sabir ${ }^{2}$, Marcus M. Keane ${ }^{1}$ \\ ${ }^{1}$ Informatics Research Unit for Sustainable Engineering, Ryan Institute, NUI Galway, Ireland \\ ${ }^{2}$ Computer Science Department, Technische Universität München \\ raymond.sterling@nuigalway.ie, struss@in.tum.de
}

\begin{abstract}
This paper presents a methodology for model-based fault detection and diagnosis underpinned by modelica models and using a qualitative approach to diagnosis, which has been applied to diagnosis of an air handling unit based on data recorded by a building management system. The main steps from model development to component diagnosis are discussed and illustrated using a heating coil component.
\end{abstract}

Keywords: model-based diagnosis, heating coil, calibration, fault detection and diagnosis

\section{Introduction}

Heating Ventilation and Air conditioning (HVAC) systems are known for being very inefficient for different reasons, one of the most common causes being the presence of undetected failures in one or more of its components. Undetected faults can remain for long periods due to different factors: compensations made by the control algorithms of other elements belonging to the same system; lack of proper maintenance, improper timing of flow of energy to/from the building, etc. Even when systems are known to suboptimal operation, the presence of faults may be very difficult to manually localize and identify, making it a costly task for human operators who only act when indoor environmental conditions are not met. This lack of timely intervention raises the need for developing automated fault detection and diagnosis methods and technologies that assist the building operator.

Different fault detection and diagnosis (FDD) methodologies have been developed for HVAC systems, mostly based on expert knowledge to help identifying the faulty condition and its source [1]. However, a new trend in FDD is that of using models of the HVAC systems providing a base line for optimal operation, and supporting the detection of deviation from this optimum [2]. Model-based methods, offer the advantage of an increased flexibility to adapt to different and innovative HVAC systems.

The focus of this paper is on a model-based diagnostic solution that uses a qualitative model for the part of the HVAC system corresponding to the Air Handling Unit (AHU). This solution is derived from a general first-principle Modelica model and exploits a general diagnosis algorithm that isolates and identifies faults that occur frequently and can cause significant loss of system performance in AHUs: passing heating- and cooling-coil valves, and stuck dampers. An application example using a heating coil model is presented and provisions are made for the extension to other components.

The paper is structured as follows: section 2 provides an intuitive introduction to model-based diagnosis (MBD); section 3 outlines model requirements for online diagnosis while section 4 presents the modelica models and its calibration. In section 5 an example of the complete tool chain is discussed and finally, sections 6 and 7 provide concluding remarks and future work.

\section{Model-Based Diagnosis: an intuitive introduction}

Models used for designing and verifying control usually capture the nominal behaviour of the controlled physical system but are less reliable when modelling behaviours related to faulty operation. In fact, modelbased diagnosis is able to perform fault localization using only models that represent the intended behaviour of the system (OK models) [3]. However, fault identification requires modelling the possible relevant faulty behaviours, as well, which may also lead to a more focused localization [3]. Therefore, for each system component, a health variable is defined as the health status (failure mode) of that component. A system health assignment (or health mode) is the set of health assignments for all components in the system.

To each component OK or failure mode, the respective (mis)behaviour is captured by a model (e.g. a set of (differential) equations. For example, a passing 


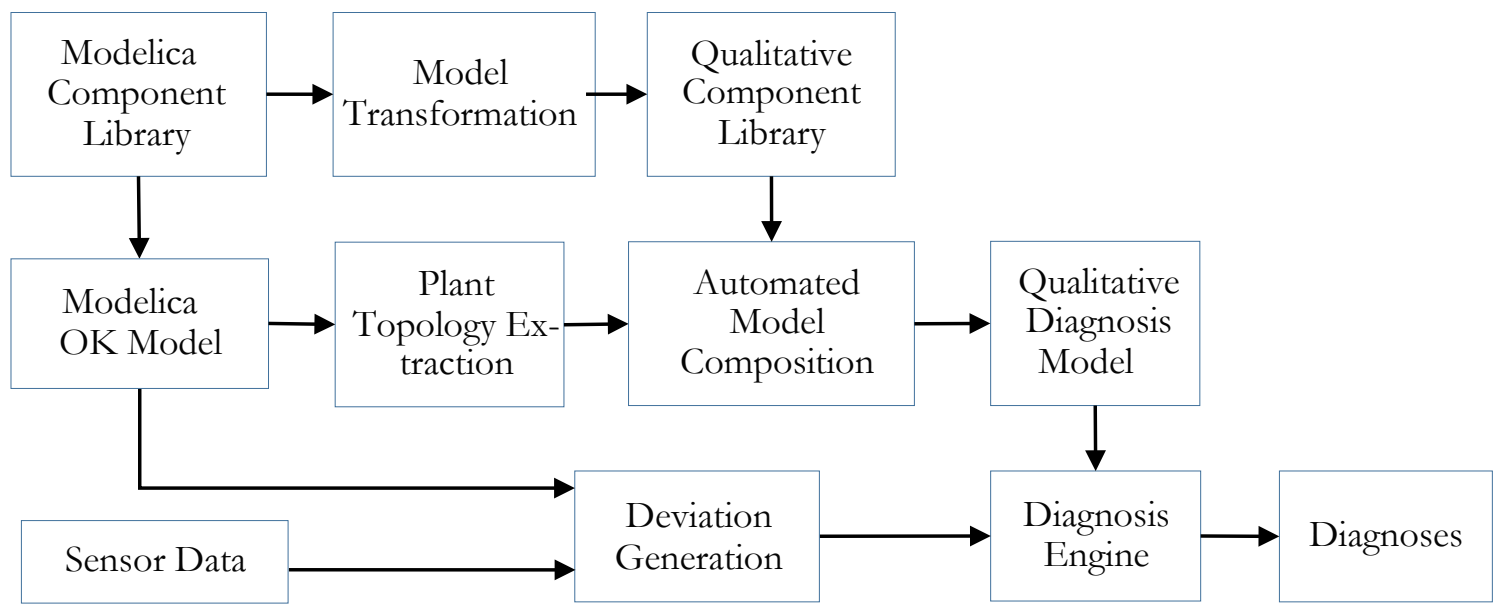

Figure 1 From model to diagnosis, the MBD chain

heating coil valve will have hot water flowing in the heating coil when the heating coil is supposed to be switched off, in which case the air heats up after passing over this heating coil. Hence, in this case, the control setting is heating coil valve closed, but with the fault, the valve is actually open.

Based on this, each system health assignment implies one behaviour model of the entire plant, which is obtained by aggregating the component (fault) models.

Model-based diagnosis is based on an explicit representation of the knowledge about the components and the information about the plant structure, which determines how the components interact with each other. Based on a library of generic component models and the representation of the plant topology, a system model (possibly covering both the nominal and faulty behaviours) can be obtained automatically. This model is exploited by a generic diagnosis algorithm, which is not plant-specific and even not domain-specific (Figure 2). This way, diagnostics tailored to a specific plant require only the specification of the plant structure and component models; they are generated automatically instead of being hand-tailored. For the purposes of this research work, a plant model consisting of component models was built manually and then fed to the diagnostics tool that produces the diagnostics system automatically. However, steps have been taken to automate the full process by parsing the modelica file. This parsing can be done as long as certain naming convention is in place.

\section{From Model to On-line Diagnosis}

In this section, we present a complete workflow and

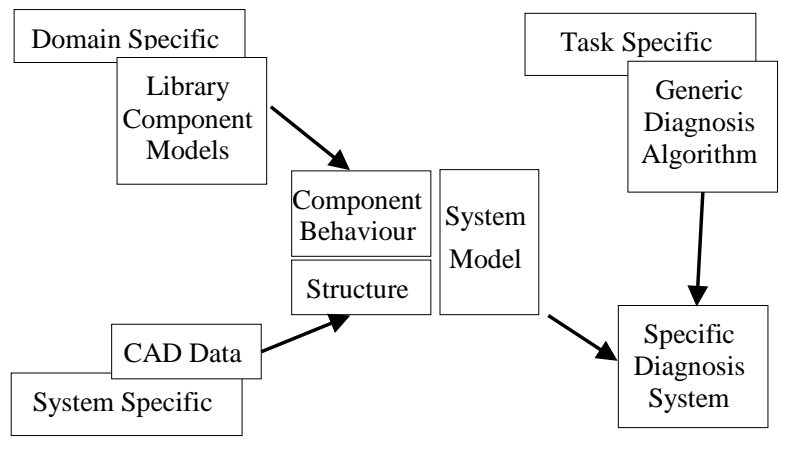

Figure 2 Generating Diagnostic Systems

system modules required to build a diagnostic solution for a class of plants (AHU) and to deploy it for a single plant and run it on-line, which is illustrated in Figure 1 . Here, we give only an overview of the steps and modules, the most important ones being discussed in more detail in the following sections.

- Producing the general solution involves:

0 the production of a library of Modelica models (section 4) and;

0 its transformation into a qualitative diagnostic model library (top row Figure 1).

- Producing an application system based on the general solution, requires

o The configuration and calibration of a Modelica model of the correct behaviour (named OK model and explained in section 4);

0 the composition of the diagnostic model based on the diagnostic library and the component structure of the plant, which can be extracted from this Modelica system model. This composition step is part of the functionality of the tool used in this work, Raz'r (from 
OCC'M Software GmbH). The extraction of the component structure from Modelica has not yet been realized, but is expected to be straightforward, given that the models have been developed following certain requirements, which are stated in section 3.1.

\section{- For on-line diagnosis,}

o qualitative deviations are generated by computing the difference between the real data (currently for steady state only) and the predictions generated by the OK model of the plant (implemented in Modelica), and determining qualitative deviations based on given thresholds. A steady state filter is used to extract steady state data from the real operation data. The resulting qualitative deviations of dependent variables (and zero deviations for the exogenous variables) are processed by

0 the runtime diagnosis engine, which is produced by the code generator from the Raz'r module by compiling the consistency-based diagnosis algorithm and the diagnostic plant model into very compact $\mathrm{C}$-code. The output is the set of all mode assignments containing minimal combinations of component faults that are consistent with the abstract observations [4].

\subsection{Requirements on Modelling for Model- Based Diagnosis}

In order to support the model-based diagnosis approach as previously outlined, the diagnosis models and, hence, also the numerical models to generate them from have to satisfy particular requirements:

- Strictly component-oriented modelling: the library has to be organized around the component types (with models that can be parameterized) that constitute the plant and that are units subject to diagnosis, e.g. heat exchangers, mass exchanger, mass movers, etc.

- Fault models should be represented (perhaps with a parameter characterizing the fault, such as the opening of a passing valve)

- The plant model has to be configured strictly according to the real physical interconnections in the plant. It must not include computational artefacts that link certain variables that are not really interacting directly via a physical connection. This includes using the concept of connectors in Modelica to reflect the channels of physical interactions between components (rather than connections via single variables as, for instance, in Matlab/Simulink).
The models in the library have to be formulated in a context-independent manner and must not rely on implicit assumptions about the presence and correct functioning of other components, even though they may exist in most standard configurations. This is relevant for two reasons: it enables the re-use of the component models for different plants, and it is a precondition for the adequacy of the models in fault situations.

\section{Modelling a Simple Heating Coil}

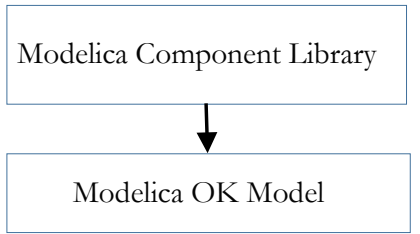

Figure 3. MBD first steps, model development and calibration

\subsection{Model Development}

Model development was driven by the specific application needs as specified in the previous section. These needs also encompass matching of the type of information interchanged between elements, reusability of the models, best use of manufacturer's data for setting up models and ease of use.

Ease of use and best use of manufacturer's data are closely related since the manufacturer's data is the first source of information a model developer will have in hand. In this regard, the developed model is such that this data is input into the parameters of the models corresponding this way to a first calibration step based on the manufacturer provided operation point. Table 1 shows the parameters, from the manufacturer's data, to be provided to the heating coil model.

Table 1. Manufacturer's datasheet operation point values needed as parameters for model setup

\begin{tabular}{|l|l|}
\hline \multirow{3}{*}{ Heating Coil } & $\begin{array}{l}\text { air input temperature } \\
\text { air output temperature } \\
\text { air mass flow rate } \\
\text { water input temperature } \\
\text { water output temperature } \\
\\
\text { water mass flow rate }\end{array}$ \\
\hline
\end{tabular}

The heating coil model calculates the outlet steadystate conditions in both, water and air sides, using equations derived from the conservation of energy and mass principles and the definition of effectiveness in the classical eff-NTU method which given by equations (1), (2) and (3) [5]: 


$$
\begin{aligned}
& Q=C_{a} *\left(T_{a O}-T_{a l}\right) \\
& Q=C_{w} *\left(T_{w l}-T_{w o}\right) \\
& Q=e^{*} f^{*} \min \left(C_{a}, C_{w}\right)^{*}\left(T_{w l}-T_{a l}\right)
\end{aligned}
$$

The effectiveness eff depends on the coil configuration (parallel flow, counter flow, or cross flow with both streams unmixed) [6]. The full modelica code is out of the scope of this paper but a snippet of the three main equations (1), (2), and (3) is shown below to illustrate the match between equation formulation and modelica code development:

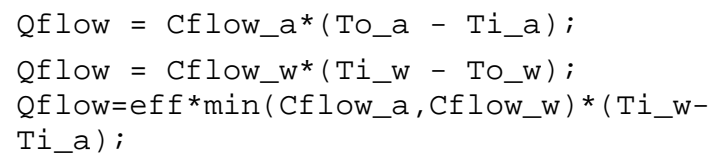

For the heating-coil component, there are inputs and outputs for flow of air through the ducting, and flow of hot water through the heating coil. Hence, massand energy-balance equations must be defined for the airflow and water-flow. The imposition of energy- and mass- balance provides the remainder of the Modelica model equations.

\subsection{Calibration}

The calibration methodology uses real operation data obtained from the facility's building management system (BMS). For the calibration procedure, instead of trying to adjust each of the component's parameters, the approach used is by assuming all the calibration can be done with the valve model explained below in this section.

In the heating coil, the air outlet temperature is controlled by water mass flow rate using valves. A control signal determines the valve's position.

Real valves have no linear behaviour but they may present non-linear behaviour and even hysteresis. To model the valve's hysteresis, several options can be followed, e.g. using on-off hysteresis, linear hysteresis and non-linear hysteresis. For the purposes of this research work, a hysteresis as shown in Figure 4 was chosen since it produced a good trade-off between accuracy and simplicity. The chosen hysteresis model will still be a good representation of the real operation of the valve while it does not add important calculation burden to the model.

There are three parameters to calibrate. ' $m$ flowMAX' is the water mass flow rate when the control signal is equal to 1 (maximum opening position), centHys and delta characterise the hysteresis' curve and the on/off points.
The real data has to be carefully observed to find maximum opening points and then the mflowMAX value is fixed in order to decrease the difference between real data and model results of the controlled variable in those points (temperature and/or humidity ratio).

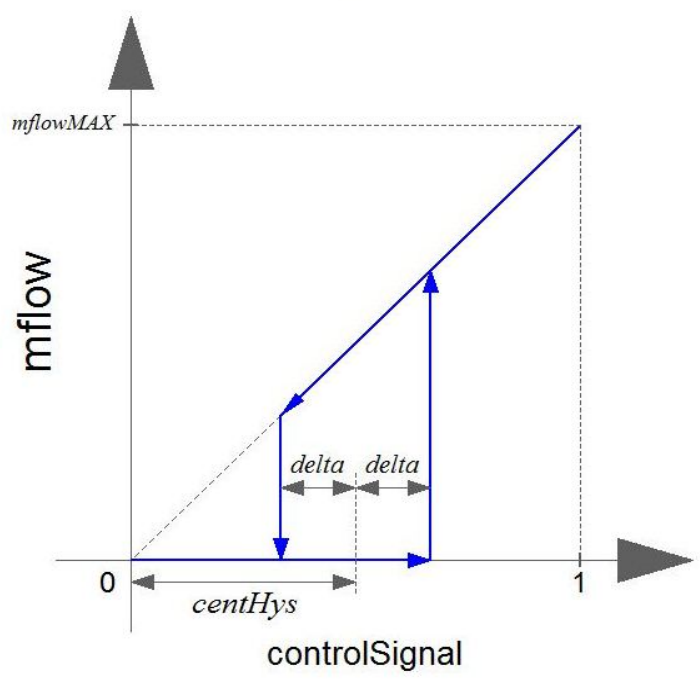

Figure 4 Valve hysteresis function

To determine centHys and delta, the employed strategy was to find sharp changes in controlled variable (output air temperature). When the controlled variable has a sharp raise, the control signal coincides with a value equal to centHys + delta; controlled variable has a sharp decrease, the control signal coincides with the value equal to centHys-delta.

Pre and post calibration results can be seen in Figure 5 and Figure 6.

In Table 2 we show calibration accuracy based on error metrics such as root mean square error (RMSE), coefficient of variation of the RMSE (CV-RMSE), mean bias error (MBE) and, normalised MBE (NMBE).

Table 2. Calibration Results

\begin{tabular}{|l|l|l|l|l|}
\hline Heating Coil & $\begin{array}{l}\text { RMSE } \\
(\mathrm{K})\end{array}$ & $\begin{array}{l}\text { CV } \\
\text { RMSE }\end{array}$ & $\begin{array}{l}\text { MBE } \\
(\mathrm{K})\end{array}$ & NMBE \\
\hline Pre-Calibration & 1.57 & 0.52 & -0.76 & -0.26 \\
\hline Pos-Calibration & 0.54 & 0.18 & -0.07 & -0.02 \\
\hline
\end{tabular}

\section{Qualitative Diagnostic Models}

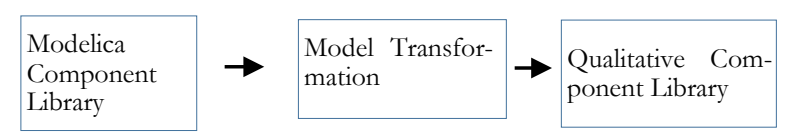

Figure 7 From numerical model to qualitative models 


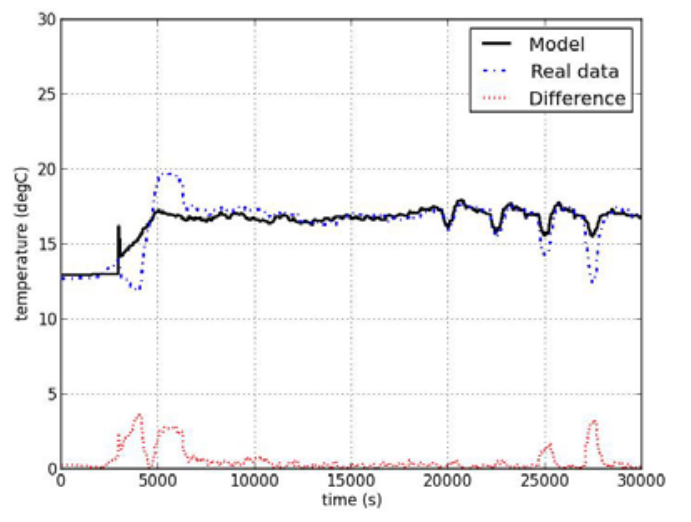

Figure 5 non-calibrated simulated (model) vs. measured (real) output air temperature for the heating coil model.

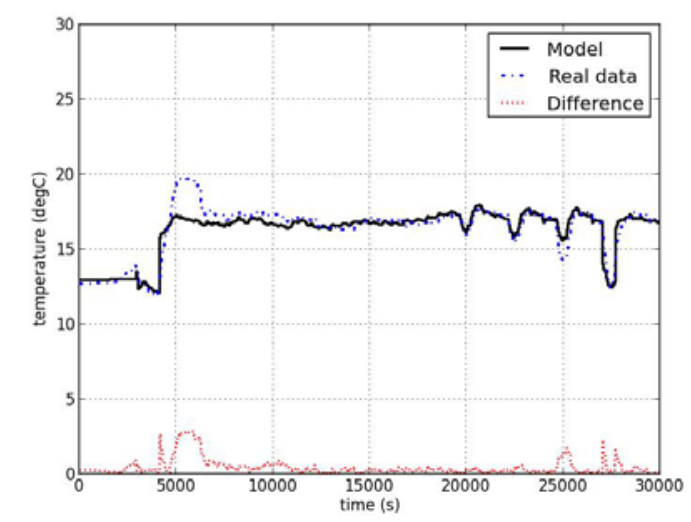

Figure 6 calibrated simulated (model) vs. measured (real) output air temperature for the heating coil model.

Creating a diagnostic library, based on the Modelica library, requires its transformation into a diagnostic model library. Figure 7 illustrates these steps.

The models used in our diagnostic approach are stated in relative, rather than absolute terms: they capture the deviation of variable values from the respective under nominal behaviour.

Following [7], [8]: the qualitative deviation of a variable $\mathrm{x}$ is defined as:

$$
\Delta \mathrm{x}:=\operatorname{sign}\left(\mathrm{x}_{\mathrm{act}}-\mathrm{x}_{\mathrm{nom}}\right)
$$

Equation (4), captures whether an actual (observed, assumed, or inferred) value is greater, less or equal to the nominal value. The latter is the value to be expected under nominal behaviour, technically: the value implied by the model in which all components are in OK mode.

Qualitative deviation models can be obtained from standard models stated in terms of (differential) equations by canonical transformations, such as equations (5) and (6). We use $\oplus, \ominus$ and $\otimes$, to denote addition, subtraction and multiplication on signs.

$$
\begin{aligned}
& a+b=c \Rightarrow \Delta a \oplus \Delta b=\Delta c \\
& a^{*} b=c \Rightarrow\left(a_{\text {act }} \otimes_{\Delta b} \oplus_{\left(b_{\text {act }}\right.}^{\otimes} \Delta a\right) \Theta_{(\Delta a} \\
& \otimes \Delta b)=\Delta c
\end{aligned}
$$

It is important to note that these equations do not contain and require values for the reference values $x_{n o m}$ and, hence, can be applied to different plants and under distinct operating modes. The qualitative deviation models, obtained from the Modelica models, reflect current modelling assumptions, (steady state, and no deviation in airflow) and become very compact due to their qualitative nature and because constants can be dropped and just replaced by their signs. Internally, this model is automatically transformed into an efficient data structure representing finite relation.

In the following, we illustrate how this transformation can be done by manipulating the equations. According to energy balance equations (equations (1), (2) and, (3)), and assuming no losses, the energy balance in equation (7) can be reformulated in terms of deviations $(\Delta)$ as in equation (8).

Assuming that the air flow and the water temperature (drop) are positive and not deviating and replacing the capacity flow by the mass flow mflow ${ }_{w}$ (which differ only by a constant factor), we obtain equation (9) which applies to all modes of the coil.

$$
\begin{aligned}
& 0=C_{a} *\left(T_{a O}-T_{a l}\right)-C_{w} *\left(T_{w l}-T_{w O}\right) \\
& 0=\Delta\left(C_{\mathrm{a}} *\left(T_{a l}-T_{a O}\right)\right) \oplus \Delta\left(C_{w} *\left(T_{w^{l}-}-T_{w o}\right)\right) \\
& 0=\Delta T_{a l} \oplus \Delta T_{\mathrm{aO}} \oplus \Delta \text { mflow }_{\mathrm{w}}
\end{aligned}
$$

Following equation (4), each of the variables used for diagnostics (equation (9)) can have a deviation of the measured value from the simulated one as follows:

- positively ('+'), when the actual (measured, predicted, or assumed) value is above the simulated plus a threshold;

- negatively ('-'), when the actual value is below the simulated minus a threshold;

- or not deviate (' 0 '), when the actual value is within the simulated value plus/minus the threshold.

Table 3 depicts the resulting relation on the three deviation variables, i.e. all solution tuples of equation (9). For instance, the first three rows of the table indicate the intuitive fact that, if the mass flow shows no deviation, a deviation of the incoming air temperature 
will simply be propagated to the output air temperature.

On the other hand, a positive deviation of the output air temperature in combination with no deviation in the input air temperature, is only consistent with a positive deviation in the mass flow rate of the water (last-but-one row). From the diagnostic perspective, this reveals a fault in the coil (e.g. a passing valve), because a correct coil will not produce a deviating water flow. A valve stuck closed may lead to a negative deviation "-“, if the command Cmd to the valve is "open” (to some non-zero position, “+”). If the control commands the valve to be shut, anyway, a stuckclosed valve would cause no deviation in the water flow. This is captured by the model fragment in Table 5 , which actually, is the complete fault model. Table 4 and Table 5 show the models of the OK mode and the passing valve, respectively. The table expresses that this mode may coincide with the nominal behaviour for a certain range of opening commands, but deviate positively for smaller valve positions.

With respect to their use for diagnosis, tables 4-6 jointly with table 3 capture which tuples of temperature and water flow deviations are consistent with which behaviour modes. Note that this does not require that the deviations can be observed directly. They may also be predicted by the system model based on observations for a particular system health assignment.

Bear in mind that a qualitative representation of one mode doesn't exclude that any other mode can be reached with the same combination of inputs/outputs.

\subsection{Runtime Deviation Generation}

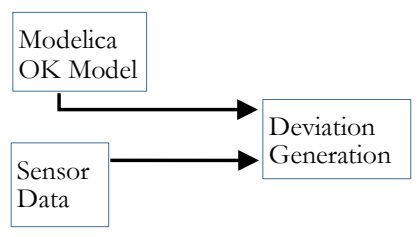

Figure 8 Generating Deviations

At runtime, the system will calculate deviations (Figure 8) by following the steps:

- Read each data vector corresponding to the sensor and actuator signals;

- Extract the exogenous variables including (external temperature, damper, and valve commands);

- Provide the exogenous variable values to the Modelica model of nominal behaviour, compare the values predicted by this model with the actual sensor data, and compute the deviations. In the current
Table 4 Qualitative representation of the OK mode

Table 3. Relation on temperature deviations and water flow deviation

\begin{tabular}{|c|c|c|c|c|}
\hline \multicolumn{3}{|c|}{ flow deviation } & 0 & 0 \\
\hline$\Delta$ mflow $_{\mathrm{w}}$ & $\Delta \mathrm{T}_{\mathrm{aI}}$ & $\Delta \mathrm{T}_{\mathrm{aO}}$ & + & 0 \\
\hline 0 & - & - & \multirow{2}{*}{\multicolumn{2}{|c|}{$\begin{array}{l}\text { Table } 5 \text { Qualitative repre } \\
\text { sentation of the stuck } \\
\text { closed valve mode }\end{array}$}} \\
\hline 0 & 0 & 0 & & \\
\hline 0 & + & + & Cmd & $\Delta$ mflow $_{\mathrm{w}}$ \\
\hline- & - & - & 0 & 0 \\
\hline - & 0 & - & + & - \\
\hline
\end{tabular}

Table 6 Qualitative representation of the passing valve mode

\begin{tabular}{|c|c|}
\hline Cmd & $\Delta$ mflow $_{\mathrm{w}}$ \\
\hline 0 & + \\
\hline+ & 0 \\
\hline+ & + \\
\hline
\end{tabular}

solution, this is simply done by using a threshold (which can be different for different variables).

For the example with the heating coil documented here, a threshold of $2^{\circ} \mathrm{C}$ was chosen in order to produce deviations in the domain of signs ('+','-','0'). In future solutions, different orders of magnitudes of the deviations could be generated by the abstraction module, which can take arbitrary sets of interval boundaries as an input.

For the example with the heating coil, Table 7 shows both the sensor data and the predicted values, highlighting the temperature before and after the heating coil. Using the $2^{\circ} \mathrm{C}$ threshold, the inflow air temperature is determined as nominal, while the outflow air temperature is higher than expected. This triggers a diagnosis event.

\subsection{Diagnosis Inference}

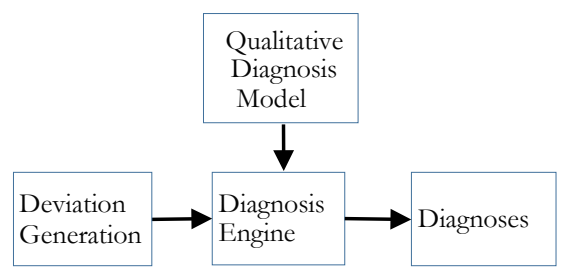

Figure 9 From deviations and qualitative model to diagnosis 
The computed deviation pattern (with a zero deviation of exogenous variables -input temperature, and valve commands-) forms the input to the diagnosis runtime system (Figure 9). The deviation patterns will be checked for consistency with the possible models. In the trivial example restricted to one component presented in Table 7, the input/output temperature deviations $(0,+)$ match with only one row in Table 3 that holds for all behaviour modes, which fixes mflow $_{\mathrm{w}}$ to be positively deviating. This positive deviation is consistent with the valve passing mode (Table 6), but neither with the OK mode, not the stuck closed mode. Note, that this result can actually be concluded without information about the command to the valve.

What is illustrated here for a single component, is actually applied to the space of plant models covered by the system health assignments, which may yield alternative diagnosis hypotheses and also such that correspond to multiple component faults.

Table 7 Deviations between sensor data and model data

\begin{tabular}{|l|c|c|}
\cline { 2 - 3 } \multicolumn{1}{c|}{} & $\mathrm{T}_{\mathrm{al}}(\mathrm{o} \mathrm{C})$ & $\mathrm{T}_{\mathrm{aO}}$ (으) \\
\hline Sensor Data & 18.32 & 20.87 \\
\hline Model Prediction & 18.44 & 18.44 \\
\hline Resulting Deviation & $\mathbf{0}$ & + \\
\hline
\end{tabular}

\section{Discussion}

In this paper a tool chain from model development to fault detection in air handling units has been presented and discussed with an illustrative example of a heating coil. The development tool of choice for the model was Modelica since it provides all the necessary tools to comply with model requirement for model-based fault detection as shown in section 3.1.

One of the main advantages of the model-based approach is the adaptability to different plants and to changes in the same plant. A brief description of the steps involved in adapting the qualitative model based diagnosis is presented below.

- Structural changes: These changes will have to be reproduced in the model, which would need to be compiled and recalibrated. The diagnosis model structure is a 1:1 mapping of the model and as such only minor adaptation is needed. However, if the change involves variables considered for diagnosis, the variable mapping between model and diagnosis framework has to be modified and tested with new data sets.

- Parameter changes: recalibration of the models is in principle the only requirement. In the case these parameter changes impact the accuracy of the model, the tolerances of the diagnosis framework might have to be adjusted.

- Sensor changes: similar consideration to the case of structural changes should be taken in the case of adding new sensors or modifying position of existing ones. In the case that existing sensors are to be replaced with new ones with different precision, the steps described in the parameter changes are to be followed.

- Changes in control: plant model and diagnosis framework is, in principle, not affected by changes in the control strategy.

This adaptability makes model-based diagnosis a viable approach to fault detection and diagnosis in air handling units.

Taking into account that heating ventilation and air conditioning systems are rarely critical systems, the benefits of FDD in the build environment are more economic and environmental rather than being a safety issue and that hourly fault detection and diagnosis frequencies are more than acceptable in building applications; there is little scope for extending the models to include dynamic behaviour at the moment. Although in early stage, there exist scope for modelica models to become the de-facto standard in energy modelling of building components as shown by the recently established International Energy Agency Annex 60 . Within this context, one of the key issues for model use during operation (e.g. Model-Based FDD, Model-Predictive Control, etc.) is the development of calibrated models that represent in a cost-effective manner the expected normal behaviour of the systems. Focused on air handling units' components, an approach to tack such problem, which can be automated, has been presented in this research paper

\section{Future Work}

Next steps in this research are:

- Development and testing of models for other components of HVAC systems;

- Improve the calibration methodology by developing an automatic calibration procedure that could be implemented underpinned by machine learning.

- Comparison of the qualitative model-based diagnosis approach with others such APAR rules or quantitative diagnosis

- Deployment and testing in a range of real units operating in normal environments. 


\section{Acknowledgements}

This work was supported by the International Energy Research Centre and Enterprise Ireland under project n. CC-2011-4005B and by the Irish Research Council - D’Appolonia enterprise partnership scheme. Special thanks to Dominik O'Sullivan John McCarthy for their invaluable support and help in providing datasets for testing the developments presented in this research work.

\section{Nomenclature}

\begin{tabular}{|c|c|c|c|}
\hline eff & & veness & \\
\hline$Q$ & & ransfer & \\
\hline C & & ty flow & \\
\hline$T$ & & rature & \\
\hline mflow & & ow rate & \\
\hline \multicolumn{4}{|c|}{ Subscripts and functions } \\
\hline$a$ & air & 1 & input \\
\hline$w$ & water & $\mathrm{O}$ & output \\
\hline $\min (\cdot, \cdot)$ & smalle & lue betu & argumen \\
\hline
\end{tabular}

\section{References}

[1] S. Katipamula, P. Michael, and R. Brambley, "Methods for Fault Detection, Diagnostics, and Prognostics for Building Systems- A Review, Part II,” HVAC\&R Res., vol. 11, no. 2, 2005.

[2] R. Isermann, "Model-based fault-detection and diagnosis - status and applications," Annu. Rev. Control, vol. 29, no. 1, pp. 71-85, Jan. 2005.

[3] P. Struss, "Model-based problem solving," in in Handbook of Knowledge Representation, Elsevier, vol. 6526, no. 07, V. L. and B. P. F. van Harmelen, Ed. Elsevier B.V., 2008, pp. $395-465$.

[4] O. Dressler and P. Struss, "The consistencybased approach to automated diagnosis of devices," in in Principles of Knowledge Representation, G. Brewka, Ed. Stanford, CA, USA: Center for the Study of Language and Information, 1996, pp. $267-311$.

[5] ASHRAE, ASHRAE Handbook:

Fundamentals (SI edition). Atlanta, GA:

\section{[oc]}

$\mathrm{g} / \mathrm{s}]$
American Society of Heating, Refrigerating and Air-conditioning Engineers, 2009.

[6] M. Wetter, "Simulation Model: Finned Water-to-Air Coil without Condensation. LBNL-42355,” 1999.

[7] P. Struss, "Models of Behavior Deviations in Model-based Systems," in European Conference on Artificial Intelligence, pp. 883-887.

[8] P. Struss and A. Fraracci, “Automated Model-Based FMEA of a Braking System,” Fault Detect. Superv. Saf. ..., no.

Safeprocess, pp. 1-6, 2012. 